Proceedings of the Edinburgh Mathematical Society (2007) 50,673-687 (C)

DOI:10.1017/S0013091504001014 Printed in the United Kingdom

\title{
INTEGRABILITY OF THE DERIVATIVE OF A BLASCHKE PRODUCT
}

\author{
DANIEL GIRELA ${ }^{1}$, JOSÉ ÁNGEL PELÁEZ² AND DRAGAN VUKOTIĆ ${ }^{3}$ \\ ${ }^{1}$ Departamento de Análisis Matemático, Universidad de Málaga, \\ Campus de Teatinos, 29071 Málaga, Spain (girela@uma.es) \\ ${ }^{2}$ Departamento de Análisis Matemático, Universidad de Sevilla, \\ Avenida de la Reina Mercedes, Apartado de correos 1160, \\ 41080 Sevilla, Spain (japelaez@us.es) \\ ${ }^{3}$ Departamento de Matemáticas, Universidad Autónoma de Madrid, \\ 28049 Madrid, Spain (dragan.vukotic@uam.es)
}

(Received 21 September 2004)

\begin{abstract}
We study the membership of derivatives of Blaschke products in Hardy and Bergman spaces, especially for the the interpolating Blaschke products and for those whose zeros lie in a Stolz domain. We obtain new and very simple proofs of some known results and prove new theorems that complement or extend the earlier works of Ahern, Clark, Cohn, Kim, Newman, Protas, Rudin, Vinogradov and others.

Keywords: Blaschke products; Hardy spaces; Bergman spaces; $Q_{p}$ spaces; Stolz angle; interpolating sequences

2000 Mathematics subject classification: Primary 30D50; 30D55; 32A36
\end{abstract}

\section{Introduction}

One of the central problems with Blaschke products is that of the membership of their derivatives in classical function spaces. This problem was studied by a number of authors in the 1970s and 1980s: for Hardy spaces $H^{p}$ by Protas [30], Ahern and Clark [3], Ahern [1] and Cohn [10]; for the related $B^{p}$ spaces, the Banach envelopes of the Hardy spaces with exponents smaller than 1 , by Ahern and Clark [3,4]; and for weighted Bergman spaces $A_{\alpha}^{p}$ by Ahern [1] and Kim [22].

The statements of many such results can be found in [11]. More recently, mean growth of the derivative of Blaschke products was investigated by Girela and González [18], Kutbi [23], and by Girela and Peláez [19]. A closely related question of the membership of Blaschke products in the $Q_{p}$ spaces, which became an active area of study in the mid and late 1990s, was investigated by Essén and Xiao [16] and Danikas and Mouratides [12], among others.

The Schwarz-Pick lemma readily implies that the derivative of any Blaschke product belongs to $\bigcap_{0<p<1} A^{p}$. Rudin [31] showed that there is a Blaschke product whose 
derivative does not belong to $A^{1}$ (see [29] for an explicit example). Ahern [2] found a necessary and sufficient condition for the membership of $B^{\prime}$ in the weighted space $A_{\alpha}^{p}$. However, his criterion is expressed in terms of $|B(z)|$ and is therefore difficult to verify in practice. It is thus desirable to have criteria for membership of $B^{\prime}$ in, say, Hardy and Bergman spaces based merely on some information on the zeros of $B$. The papers cited mainly consider conditions on the moduli of the zeros, as is the case in $[\mathbf{2 2}, \mathbf{3 0}]$. Many results obtained there appear to be sharp but the sharpness issue was not discussed.

In this paper we essentially determine the best possible exponents of integrability, either with respect to the area measure in the disc or with respect to the normalized arc length measure on the unit circle of $B^{\prime}$ for those Blaschke products $B$ which are either interpolating or have their zeros in a Stolz angle (or both). We obtain several new results and very simple proofs of some other, essentially known, results. Besides our own new techniques, we also employ those used earlier by Ahern, Clark, Naftalevich and Vinogradov, among others.

Ahern and Clark [3] considered the condition that the zeros of $B$ converge to 1 nontangentially (which is equivalent to requiring that the zeros belong to a Stolz angle) in $[\mathbf{3}, \S 4]$, again in conjunction with a condition on the moduli. By putting together various theorems from different sections of $[\mathbf{3}]$, we can readily deduce the following result: if the zeros of $B$ belong to a Stolz angle, then $B^{\prime} \in \bigcap_{0<p<1 / 2} H^{p}$ and the exponent $\frac{1}{2}$ is sharp. In our view, the statement in this form deserves to be better known, and so we formulate it and give a new and very short proof in $\S 2$.

By a theorem of Hardy and Littlewood, $H^{p} \subset A^{2 p}$ and the exponent $2 p$ cannot be improved (see $[\mathbf{1 3}$, Theorem 5.6], $[\mathbf{2 4}]$ or $[\mathbf{3 4}]$ for a simple proof). It is, thus, natural to ask whether the exponent one in the inclusion $B^{\prime} \in \bigcap_{0<p<1} A^{p}$ is sharp if the zeros of $B$ converge nontangentially to a point in the unit circle. However, a rather surprising phenomenon occurs here: $B^{\prime} \in \bigcap_{0<p<3 / 2} A^{p}$, whenever the zeros of $B$ lie in a Stolz angle. Though this can easily be deduced from Ahern's paper [1], we give a proof based only on the Schwarz lemma and the fact that $B^{\prime} \in \bigcap_{0<p<1 / 2} H^{p}$. This is also part of $\S 2$. Our findings thus provide plenty of explicit examples of functions in a Hardy space that belong to a Bergman space with approximately the triple exponent of integrability.

We devote $\S 3$ to the interpolating Blaschke products. This is the term commonly used for those products whose zero sequences are uniformly separated (equivalently, interpolating for $\left.H^{\infty}\right)$. For such products $B$ and $1<p<2$ we show that $B^{\prime} \in A^{p}$ if and only if $\sum_{n}\left(1-\left|a_{n}\right|\right)^{2-p}<\infty$. This is the converse of a theorem due to Kim [22] in this situation. In the same section, we prove the existence of an interpolating Blaschke product whose derivative does not belong to $A^{p}$ for any $p>1$. Nonetheless, for those interpolating Blaschke products whose zeros lie in a Stolz angle, we show that their derivative belongs to all $H^{p}$ spaces with $p<1$ and to all $A^{p}$ spaces with $p<2$, in accord with the usual pattern. Originally, we proved this statement using results of Danikas and Mouratides [12] and of Essén and Xiao [16] about the sequences of zeros of functions in $Q_{p}$ spaces, $0<p<1$. Later we discovered that this can be deduced from the fact that an interpolating sequence in a Stolz angle is a finite union of exponential sequences, which is implicit in the proof of [26, Theorem 3]. 


\section{Blaschke products with zeros in a Stolz angle}

We consider the standard Hardy spaces $H^{p}$ of analytic functions $f$ in the unit disc $\mathbb{D}$ for which the Hardy-Littlewood integral means

$$
M_{p}(r, f)=\left(\int_{0}^{2 \pi}\left|f\left(r \mathrm{e}^{\mathrm{i} \theta}\right)\right|^{p} \frac{\mathrm{d} \theta}{2 \pi}\right)^{1 / p}
$$

remain bounded as $r \rightarrow 1^{-}$. The reader is referred to [13] or [17] for the basic theory of these spaces. If a sequence of points $\left\{a_{n}\right\}$ in $\mathbb{D}$ satisfies the Blaschke condition: $\sum_{n=1}^{\infty}(1-$ $\left.\left|a_{n}\right|\right)<\infty$, the corresponding Blaschke product $B$ is defined as

$$
B(z)=\prod_{n=1}^{\infty} \frac{\left|a_{n}\right|}{a_{n}} \frac{a_{n}-z}{1-\bar{a}_{n} z} .
$$

Such a product is analytic in $\mathbb{D}$, bounded by 1 , and has radial limits of modulus 1 almost everywhere on the unit circle.

\subsection{Membership of the derivative in Hardy spaces}

There are several ways of saying that a point belongs to a Stolz angle. We will adopt the following one. Given $\xi \in \partial \mathbb{D}$ (the unit circle) and $\sigma \in[1, \infty)$ we set

$$
\Omega_{\sigma}(\xi)=\{z \in \mathbb{D}:|1-\bar{\xi} z| \leqslant \sigma(1-|z|)\} .
$$

Any such domain $\Omega_{\sigma}(\xi), 1 \leqslant \sigma<\infty$, will be called a Stolz angle with vertex at $\xi$. The domain $\Omega_{\sigma}(1)$ will be denoted simply by $\Omega_{\sigma}$. The following observation, which can be found in [33], will be useful in what follows:

$$
\frac{1}{2+\sigma} \leqslant \frac{|1-\bar{\lambda} z|}{|1-| \lambda|z|} \leqslant 2+\sigma, \quad \text { whenever } z \in \mathbb{D}, \lambda \in \Omega_{\sigma}
$$

Lemma 2.1. Given $\sigma \in[1, \infty)$, there exists a constant $K_{\sigma}>0$ such that, if $a \in \Omega_{\sigma}$, then

$$
\left|\frac{1-z}{1-\bar{a} z}\right| \leqslant K_{\sigma} \quad \text { for every } z \in \mathbb{D} \text {. }
$$

Proof. We first consider the case when $a$ is real and $0<a<1$. Set

$$
S(z)=\frac{1-z}{1-a z}, \quad z \in \mathbb{D}
$$

Bearing in mind that $S$ is a Möbius transformation with real coefficients, we easily see that $S(\mathbb{D})$ is the disc with centre $1 /(1+a)$ and radius $1 /(1+a)$ and is therefore contained in the disc of radius $2 /(1+a)$ centred at the origin. Thus, we have

$$
\left|\frac{1-z}{1-a z}\right| \leqslant \frac{2}{1+a} \leqslant 2, \quad z \in \mathbb{D}, 0<a<1 .
$$


Now, for arbitrary $a$ in $\Omega_{\sigma}$, using (2.2) and the special case just considered, we obtain

$$
\left|\frac{1-z}{1-\bar{a} z}\right|=\left|\frac{1-z}{1-|a| z}\right|\left|\frac{1-|a| z}{1-\bar{a} z}\right| \leqslant 2(2+\sigma) .
$$

Hence, we can choose $K_{\sigma}=2(2+\sigma)$.

Ahern and Clark [3, Lemma 1, p. 121] found a practical criterion for membership of the derivative of a Blaschke product in a Hardy space.

Lemma 2.2. If a Blaschke product $B$ has zeros $a_{n}=r_{n} \mathrm{e}^{\mathrm{i} t_{n}}$, then $B^{\prime} \in H^{p}$ if and only if the function $f$ defined on the unit circle by

$$
f(t)=\sum_{n=1}^{\infty} \frac{1-\left|a_{n}\right|}{\left(1-\left|a_{n}\right|\right)^{2}+\left(t-t_{n}\right)^{2}}
$$

belongs to $L^{p}(0,2 \pi)$.

We now combine the above lemmas to give a simple proof of a theorem on integrability of the derivative of Blaschke products with zeros in a Stolz angle. The result is due to Ahern and Clark $[\mathbf{3}]$ although it was not stated there in a unified way. Part (a) follows from [3, Theorem 12] in the case $\alpha=\gamma=1$, with minor modifications of the domain $R(\delta, \gamma)$. Part (b) is contained in part (ii) of [3, Theorem 8].

Our proof is based on a simple and direct idea. Many complex analysts are familiar with the following exercise: if the zeros of a Blaschke product $B$ lie on the radius $(0,1)$ and $f(z)=(1-z)^{2} B(z)$, then $f^{\prime}$ is bounded in the unit disc (see, for example, [32, Exercise 18, Chapter 15]). This gives some basic information about the growth of $B^{\prime}$ as $z \rightarrow 1$ along the radius $[0,1)$. With the aid of Lemma 2.1 we can generalize the idea used above to a Stolz angle to obtain the proof of part (a) of the theorem below.

\section{Theorem 2.3.}

(a) If the zeros of a Blaschke product $B$ all lie in some Stolz angle, then $B^{\prime} \in$ $\bigcap_{0<p<1 / 2} H^{p}$.

(b) The Blaschke product $B$ with zeros $a_{n}=1-1 /\left(n \log ^{2} n\right), n \geqslant 2$, has the property that $B^{\prime} \notin H^{1 / 2}$.

Proof of Theorem 2.3. (a) Without loss of generality we may assume that the Stolz angle has its vertex at $z=1$. Since the function given by $f(z)=(1-z)^{-2}$ is a member of $H^{p}$ whenever $p<\frac{1}{2}$, it suffices to show that

$$
\left|B^{\prime}(z)\right| \leqslant \frac{C}{|1-z|^{2}}, \quad \text { whenever } z \in \mathbb{D},
$$

for some universal constant $C$.

Denoting by $a_{n}$ the zeros of $B$, let us agree to write

$$
b_{n}(z)=\frac{\left|a_{n}\right|}{a_{n}} \frac{a_{n}-z}{1-\bar{a}_{n} z}, \quad B(z)=\prod_{n=1}^{\infty} b_{n}(z), \quad B_{n}(z)=\frac{B(z)}{b_{n}(z)} .
$$


Then

$$
B^{\prime}(z)=\sum_{n=1}^{\infty} b_{n}^{\prime}(z) B_{n}(z)
$$

whence

$$
\left|B^{\prime}(z)\right| \leqslant \sum_{n=1}^{\infty} \frac{1-\left|a_{n}\right|^{2}}{\left|1-\bar{a}_{n} z\right|^{2}}\left|B_{n}(z)\right| .
$$

Applying Lemma 2.1, we obtain

$$
\left|(1-z)^{2} B^{\prime}(z)\right| \leqslant \sum_{n=1}^{\infty}\left(1-\left|a_{n}\right|^{2}\right)\left|\frac{1-z}{1-\bar{a}_{n} z}\right|^{2} \leqslant 2 K_{\sigma}^{2} \sum_{n=1}^{\infty}\left(1-\left|a_{n}\right|\right) .
$$

The statement follows since $\left\{a_{n}\right\}$ is a Blaschke sequence by assumption.

(b) We use Lemma 2.2. In this case all $t_{n}=0$; hence, the function $f$ becomes

$$
f(t)=\sum_{n=2}^{\infty} \frac{1 /\left(n \log ^{2} n\right)}{1 /\left(n^{2} \log ^{4} n\right)+t^{2}}=\sum_{n=2}^{\infty} \frac{n \log ^{2} n}{1+t^{2} n^{2} \log ^{4} n} .
$$

For $t>0$, let $N_{t}$ be the unique number greater than 1 with the property that

$$
t N_{t} \log ^{2} N_{t}=1
$$

Then we have

$$
f(t) \geqslant \sum_{2 \leqslant n \leqslant N_{t}} n \log ^{2} n \asymp N_{t}^{2} \log ^{2} N_{t}
$$

by a standard argument involving summation by parts. Thus,

$$
f(t)^{1 / 2} \geqslant C N_{t} \log N_{t}=\frac{C}{t \log N_{t}}
$$

for a fixed positive constant $C$. Recalling the definition of $N_{t}$, we see that

$$
\log N_{t}+2 \log \log N_{t}=\log \frac{1}{t}
$$

and it then follows that $\log N_{t} \sim \log (1 / t)$, as $t \rightarrow 0$. Consequently, there exist positive constants $\alpha$ and $t_{0}$ such that $f(t)^{1 / 2} \geqslant \alpha /(t \log (1 / t))$ for all $t \in\left[0, t_{0}\right]$. Therefore,

$$
\int_{0}^{2 \pi} f(t)^{1 / 2} \mathrm{~d} t \geqslant \alpha \int_{0}^{t_{0}} \frac{1}{t \log (1 / t)} \mathrm{d} t=\infty
$$

so $B^{\prime} \notin H^{1 / 2}$ by Lemma 2.2 .

Note that the same exponent also seemed critical under other assumptions on the zeros considered in $[\mathbf{3}, \mathbf{2 2}, \mathbf{3 0}]$. 


\subsection{Membership of the derivative in Bergman spaces}

Denote by $\mathrm{d} A$ the normalized Lebesgue area measure on the disc,

$$
\mathrm{d} A(z)=\pi^{-1} r \mathrm{~d} r \mathrm{~d} \theta=\pi^{-1} \mathrm{~d} x \mathrm{~d} y,
$$

and by $L^{p}(\mathrm{~d} A)$ the standard Lebesgue space equipped with the usual norm or metric, depending on whether $1 \leqslant p<\infty$ or $0<p<1$. The Bergman space $A^{p}$ is the closed subspace of $L^{p}(\mathrm{~d} A)$ consisting of analytic functions in $\mathbb{D}$. We refer the reader to $[\mathbf{7 , 1 4 , 2 1}]$ for the theory of these spaces.

Our goal in this subsection is to obtain a result analogous to part (a) of Theorem 2.3 for Bergman spaces.

By a holomorphic self-map of the disc we mean an analytic function $\varphi$ in $\mathbb{D}$ for which $\varphi(\mathbb{D}) \subset \mathbb{D}$. The following simple statement based on the Schwarz-Pick lemma will be useful throughout the paper.

Proposition 2.4. Let $\varphi$ be an arbitrary holomorphic self-map of the disc. If $\varphi^{\prime} \in H^{p}$, then $\varphi^{\prime} \in A^{p+1-\varepsilon}$ for any positive and sufficiently small $\varepsilon$.

Proof. By the Schwarz-Pick lemma we have

$$
\begin{aligned}
\int_{\mathbb{D}}\left|\varphi^{\prime}(z)\right|^{p+1-\varepsilon} \mathrm{d} A(z) & \leqslant \int_{\mathbb{D}}\left|\varphi^{\prime}(z)\right|^{p}\left(\frac{1-|\varphi(z)|^{2}}{1-|z|^{2}}\right)^{1-\varepsilon} \mathrm{d} A(z) \\
& \leqslant \int_{\mathbb{D}}\left|\varphi^{\prime}(z)\right|^{p}\left(\frac{1}{1-|z|}\right)^{1-\varepsilon} \mathrm{d} A(z) \\
& =2 \int_{0}^{1} M_{p}^{p}\left(r, \varphi^{\prime}\right) \frac{r}{(1-r)^{1-\varepsilon}} \mathrm{d} r \\
& \leqslant 2\left\|\varphi^{\prime}\right\|_{H^{p}}^{p} \int_{0}^{1} \frac{1}{(1-r)^{1-\varepsilon}} \mathrm{d} r \\
& =\frac{2}{\varepsilon}\left\|\varphi^{\prime}\right\|_{H^{p}}^{p},
\end{aligned}
$$

and the statement follows.

Theorem 2.3 and Proposition 2.4 immediately yield the following slightly surprising result.

Theorem 2.5. If the zeros of a Blaschke product $B$ all lie in some Stolz angle, then $B^{\prime} \in \bigcap_{0<p<3 / 2} A^{p}$.

One usually encounters the following types of examples regarding the membership in Hardy and Bergman spaces:

(i) lacunary series that belong to many (or all) Bergman spaces, but do not lie in any Hardy space; or

(ii) functions that follow the usual pattern of belonging to $H^{p}$ for $p<p_{0}$ and to $A^{q}$ when $q<2 p_{0}$; for example, this happens with the appropriate negative powers of $1-z$ and with conformal maps onto angular domains. 
Theorem 2.5 can also be deduced from [1, Theorem 6.1] and Theorem 2.3 by means of the Schwarz-Pick lemma and integration in $\theta$, and the proof works even for arbitrary inner functions. In summary, our contribution here consists in giving an even simpler proof using Proposition 2.4. This explains why we have $\frac{3}{2}$ instead of simply 1 in the exponent. Yet another proof, inspired by ideas from [33], can be found in [28].

Is is natural to ask whether or not the exponent $\frac{3}{2}$ is sharp. Interpolating Blaschke products do not help here. Indeed, the following result shows that if a counterexample for the exponent $\frac{3}{2}$ in Theorem 2.5 can be constructed, it cannot be obtained by using interpolating sequences.

Theorem 2.6. Let $B$ be an interpolating Blaschke product whose zero sequence lies in a Stolz angle. Then

(i) $B^{\prime} \in H^{p}$ for all $p \in(0,1)$,

(ii) $B^{\prime} \in A^{p}$ for all $p \in(0,2)$.

This is one of the results that we shall prove in the next section (which is mainly devoted to interpolating Blaschke products).

\section{Derivatives of interpolating Blaschke products}

In order to see where we stand in a general situation, let us recall two results due to Protas and Cohn, respectively.

Protas [30] studied the membership of general Blaschke products in Hardy spaces in terms of their zero sequences and proved the following.

Theorem 3.1. Whenever $\left\{a_{n}\right\}$ is the zero set of a Blaschke product $B$ and

$$
\sum_{n=1}^{\infty}\left(1-\left|a_{n}\right|\right)^{1-p}<\infty, \quad \frac{1}{2}<p<1,
$$

we have $B^{\prime} \in H^{p}$.

For a generalization, see $[\mathbf{1}$, Theorem 6.2]. Cohn [10] proved the converse of Theorem 3.1 for interpolating Blaschke products. This will be discussed in $\S 3.2$.

The following result is due to Kim [22].

Theorem 3.2. Let $\left\{a_{n}\right\}$ be the zero set of a Blaschke product $B$ and suppose also that

$$
\sum_{n=1}^{\infty}\left(1-\left|a_{n}\right|\right)^{2-p}<\infty, \quad 1<p<2
$$

Then $B^{\prime} \in A^{p}$.

The theorems above have the typical flavour of most results that appear in the literature when only the absolute values of the zeros are taken into account. Imposing additional conditions helps to improve the exponent of integrability of the derivative in most situations; that is precisely the leitmotif of this article (and many others already published on this topic).

We will show that Theorem 3.2 also has a converse for interpolating sequences. 


\subsection{Background on interpolating sequences}

We first review some background on interpolating sequences. Throughout this section, $\varrho$ will denote the pseudo-hyperbolic metric in the unit disc:

$$
\varrho(z, w)=\left|\frac{z-w}{1-\bar{w} z}\right|, \quad z, w \in \mathbb{D} .
$$

The Schwarz-Pick lemma tells us that $\varrho(f(z), f(w)) \leqslant \varrho(z, w)$ whenever $f$ is a holomorphic self-map of $\mathbb{D}$ and $z, w \in \mathbb{D}$. Also, equality holds whenever $f$ is a disc automorphism, i.e. $\varrho$ is invariant under the conformal maps of the disc onto itself.

A pseudo-hyperbolic disc of (pseudo-hyperbolic) centre $a$ and radius $r(a \in \mathbb{D}, 0<r<$ $1)$ is the set $\Delta(a, r)=\{z \in \mathbb{D}: \varrho(a, z)<r\}$. It coincides with the Euclidean disc whose (Euclidean) radius and centre are $[\mathbf{7}]$, respectively, given by

$$
R=\frac{1-|a|^{2}}{1-r^{2}|a|^{2}} r, \quad c=\frac{1-r^{2}}{1-r^{2}|a|^{2}} a .
$$

A sequence $\left\{a_{n}\right\}$ of points in $\mathbb{D}$ is said to be uniformly separated if it satisfies the condition

$$
\inf _{n} \prod_{m \neq n} \varrho\left(a_{m}, a_{n}\right)=\inf _{n} B_{n}\left(a_{n}\right) \geqslant \delta>0
$$

(using our earlier notation $B_{n}=B / b_{n}$ as in (2.4)). By a celebrated theorem of Carleson $\left[\mathbf{8}\right.$, Theorem 3] (see also [13, Chapter 9]), $\left\{a_{n}\right\}$ is an interpolating sequence for the space $H^{\infty}$ of bounded analytic functions in the disc if and only if it satisfies (3.4). The Blaschke products whose zeros obey this condition are denominated interpolating Blaschke products and constitute an important and much studied class of Blaschke products.

\subsection{Interpolating Blaschke products}

Cohn [10] proved the following converse of Protas's theorem (Theorem 3.1) for interpolating sequences.

Theorem 3.3. If $\left\{a_{n}\right\}$ is the zero set of an interpolating Blaschke product $B$ such that $B^{\prime} \in H^{p}, \frac{1}{2}<p<1$, then

$$
\sum_{n=1}^{\infty}\left(1-\left|a_{n}\right|\right)^{1-p}<\infty .
$$

The following statement shows that Kim's theorem (Theorem 3.2) allows for a converse in the case of interpolating sequences. We use the notation ' $\gtrsim$ ' to signify that one positive quantity is greater than another times a fixed constant and ' $\asymp$ ' to signify that the quotient of two positive quantities is bounded from above and away from zero.

Theorem 3.4. If $B$ is an interpolating Blaschke product with zeros $\left\{a_{n}\right\}$, then

$$
\int_{\mathbb{D}}\left|B^{\prime}(z)\right|^{p} \mathrm{~d} A(z) \gtrsim \sum_{n=1}^{\infty}\left(1-\left|a_{n}\right|\right)^{2-p} .
$$

In particular, if the series on the right diverges, then $B^{\prime} \notin A^{p}$. 
This theorem is the converse of [22, Theorem 3.1]. The statement is non-trivial only when $1<p<2$. Of course, it adds nothing new when $0<p \leqslant 1$ because we already have the Blaschke condition. On the other hand, we know that if $B$ is an arbitrary infinite Blaschke product, then $B^{\prime} \notin A^{p}$ for any $p \geqslant 2$ (see [22, Theorem 1.1]).

In order to prove Theorem 3.4 we need the following lemma.

Lemma 3.5. Let $\left\{a_{n}\right\}_{n=1}^{\infty}$ be a uniformly separated sequence of points in the unit disc $\mathbb{D}$ with the constant $\delta$ as in (3.4), and let $B$ be the Blaschke product whose sequence of zeros is $\left\{a_{n}\right\}_{n=1}^{\infty}$. There then exist two positive constants, $\alpha$ and $\beta$, that depend only on $\delta$ such that the pseudo-hyperbolic discs $\left\{\Delta\left(a_{n}, \alpha\right)\right\}_{n=1}^{\infty}$ are pairwise disjoint and

$$
\left|B^{\prime}(z)\right| \geqslant \frac{\beta}{1-\left|a_{n}\right|}, \quad \text { for all } z \in \Delta\left(a_{n}, \alpha\right), n=1,2, \ldots
$$

Proof. Let $b_{n}$ and $B_{n}=B / b_{n}$ be defined as in (2.4). Using (3.4), the definition of $\varrho$, the triangle inequality and the Schwarz-Pick lemma applied to the self-map $B_{n}$ of $\mathbb{D}$, we obtain

$$
\begin{aligned}
\delta & \leqslant\left|B_{n}\left(a_{n}\right)\right| \\
& =\varrho\left(B_{n}\left(a_{n}\right), 0\right) \\
& \leqslant \varrho\left(B_{n}\left(a_{n}\right), B_{n}(z)\right)+\varrho\left(B_{n}(z), 0\right) \\
& \leqslant \varrho\left(a_{n}, z\right)+\left|B_{n}(z)\right|,
\end{aligned}
$$

for all $z \in \mathbb{D}$. It follows that

$$
\left|B_{n}(z)\right| \geqslant \frac{1}{2} \delta, \quad \text { whenever } z \in \Delta\left(a_{n}, \frac{1}{2} \delta\right) .
$$

(Note that a very similar proof of the above formula can be found in [27, p. 1800].)

Continuing with the proof of the lemma, we have

$$
\begin{aligned}
\left|B^{\prime}(z)\right| & =\left|b_{n}^{\prime}(z) B_{n}(z)+b_{n}(z) B_{n}^{\prime}(z)\right| \\
& \geqslant\left|b_{n}^{\prime}(z)\right|\left|B_{n}(z)\right|-\left|b_{n}(z)\right|\left|B_{n}^{\prime}(z)\right| .
\end{aligned}
$$

Now,

$$
\left|b_{n}^{\prime}(z)\right|=\frac{1-\left|a_{n}\right|^{2}}{\left|1-\bar{a}_{n} z\right|^{2}}=\frac{1-\varrho\left(z, a_{n}\right)^{2}}{1-|z|^{2}} \geqslant \frac{1-\varrho\left(z, a_{n}\right)}{1-|z|^{2}} .
$$

Also, by the Schwarz-Pick lemma applied to $B_{n}$,

$$
\left|B_{n}^{\prime}(z)\right| \leqslant \frac{1}{1-|z|^{2}}, \quad z \in \mathbb{D} .
$$

Together with (3.8), (3.9) and (3.7), this gives

$$
\left|B^{\prime}(z)\right| \geqslant \frac{1}{1-|z|^{2}}\left(\left(1-\frac{\delta}{2}\right) \frac{\delta}{2}-\frac{\delta}{4}\right) \geqslant \frac{\delta(1-\delta)}{4\left(1-|z|^{2}\right)} \geqslant \frac{\delta(1-\delta)}{8(1-|z|)},
$$

whenever $z \in \Delta\left(a_{n}, \frac{1}{4} \delta\right)$. 
Set $\alpha=\frac{1}{4} \delta$. Bearing in mind that $\Delta\left(a_{n}, \alpha\right)$ is the Euclidean disc with centre

$$
\frac{1-\alpha^{2}}{1-\alpha^{2}\left|a_{n}\right|^{2}} a_{n}
$$

and radius

$$
\frac{1-\left|a_{n}\right|^{2}}{1-\alpha^{2}\left|a_{n}\right|^{2}} \alpha
$$

an easy calculation gives

$$
1-|z| \leqslant \frac{4}{1-\alpha}\left(1-\left|a_{n}\right|\right), \quad z \in \Delta\left(a_{n}, \alpha\right),
$$

and then (3.10) implies that

$$
\left|B^{\prime}(z)\right| \geqslant \frac{\delta(1-\delta)(1-\alpha)}{32} \frac{1}{1-\left|a_{n}\right|}, \quad z \in \Delta\left(a_{n}, \alpha\right) .
$$

The sequence $\left\{a_{n}\right\}$ satisfies

$$
\varrho\left(a_{m}, a_{n}\right) \geqslant \delta, \quad \text { whenever } m \neq n \text {. }
$$

Since $\alpha<\frac{1}{2} \delta$, the triangle inequality implies that the pseudo-hyperbolic discs $\Delta\left(a_{n}, \alpha\right)$ are pairwise disjoint. Hence, the lemma is proved with

$$
\alpha=\frac{1}{4} \delta \quad \text { and } \quad \beta=\frac{1}{32} \delta(1-\delta)(1-\alpha) .
$$

Proof of Theorem 3.4. Let $\delta$ be the constant from the uniform separation condition (3.4). Then the pseudo-hyperbolic discs $\Delta\left(a_{n}, \alpha\right)$ chosen as in Lemma 3.5 are pairwise disjoint. Denote by $R_{k}$ the Euclidean radius of such a disc. Then

$$
R_{k}=\frac{1-\left|a_{n}\right|^{2}}{1-\alpha^{2}\left|a_{n}\right|^{2}} \alpha \asymp 1-\left|a_{n}\right| \quad \text { as } n \rightarrow \infty .
$$

Using the property that the discs $\Delta\left(a_{n}, \alpha\right)$ are pairwise disjoint, together with inequality (3.6) and asymptotic relation (3.11), we obtain

$$
\begin{aligned}
\int_{\mathbb{D}}\left|B^{\prime}\right|^{p} \mathrm{~d} A & \geqslant \sum_{n=1}^{\infty} \int_{\Delta\left(a_{n}, \alpha\right)}\left|B^{\prime}\right|^{p} \mathrm{~d} A \\
& \gtrsim \sum_{n=1}^{\infty}\left(\frac{\beta}{1-\left|a_{n}\right|}\right)^{p} R_{k}^{2} \\
& \asymp \sum_{n=1}^{\infty}\left(1-\left|a_{n}\right|\right)^{2-p}
\end{aligned}
$$

and we are done. 
From Kim's theorem (Theorem 3.2) and Theorem 3.4 we easily derive the following characterization.

Corollary 3.6. Let $\left\{a_{n}\right\}$ be an interpolating sequence, let $B$ be the corresponding Blaschke product, and let $1<p<2$. Then $B^{\prime} \in A^{p}$ if and only if

$$
\sum_{n=1}^{\infty}\left(1-\left|a_{n}\right|\right)^{2-p}<\infty .
$$

\subsection{Exponential zero sequences versus other interpolating sequences}

We wish to compare the results about integrability of the derivative of Blaschke products from three distinguished classes.

The first class is that of exponential sequences. Recall that $\left\{a_{n}\right\}$ is said to be an exponential sequence in $\mathbb{D}$ if

$$
1-\left|a_{n+1}\right| \leqslant q\left(1-\left|a_{n}\right|\right)
$$

for some fixed $q, 0<q<1$, and all $n$. As is well known, every exponential sequence is interpolating, i.e. uniformly separated (see [13, Chapter 9]).

Kim $[\mathbf{2 2}]$ proved that if the zeros of $B$ form an exponential sequence, then $B^{\prime} \in$ $\bigcap_{0<p<2} A^{p}$. Actually, using Theorem 3.1 we can deduce the stronger result: $B^{\prime} \in$ $\bigcap_{0<p<1} H^{p}$. This argument leads to a proof of Theorem 2.6.

Proof of Theorem 2.6. Let $B$ be an interpolating Blaschke product whose sequence of zeros $\left\{a_{n}\right\}_{n=1}^{\infty}$ is contained in a Stolz angle. Using a result of Newman [26, p. 506], we deduce that $\left\{a_{n}\right\}_{n=1}^{\infty}$ is a finite union of exponential sequences. It then follows that $\sum_{n=1}^{\infty}\left(1-\left|a_{n}\right|\right)^{p}<\infty$, for all positive $p$. Then Protas's theorem (Theorem 3.1) shows that $B^{\prime} \in \bigcap_{0<p<1} H^{p}$. This is (a). The inclusion $H^{p} \subset A^{2 p}(0<p<\infty)$ yields (b).

We should point out that the exponents 1 and 2 in Theorem 2.6 are both sharp. We have already seen this for $A^{p}$ spaces; for $H^{p}$ spaces this follows either from the inclusion $H^{p} \subset A^{2 p}$ or from a classical theorem of Privalov [13, Theorem 3.11].

It is a matter of simple calculus to check that every exponential sequence satisfies the condition

$$
\sum_{n=1}^{\infty}\left(1-\left|a_{n}\right|\right) \log \frac{1}{1-\left|a_{n}\right|}<\infty,
$$

which has also been considered by various authors. For example, Rudin [31] proved that if the zeros of $B$ satisfy condition (3.13), then $B^{\prime} \in A^{1}$.

The following very useful theorem is due to Naftalevich [25]. Even though it is almost 50 years old and is mentioned in [11] and listed as an exercise in [17], it does not seem to be widely used. The original paper $[\mathbf{2 5}]$ is not easily accessible, but the reader can find a detailed proof in $[\mathbf{9}]$.

Theorem 3.7. For any Blaschke sequence $\left\{a_{n}\right\}$, there exists an interpolating sequence $\left\{z_{n}\right\}$ such that $\left|z_{n}\right|=\left|a_{n}\right|$ for each $n$. 
Note that there are interpolating sequences that do not satisfy (3.13). This can be seen using Naftalevich's theorem (Theorem 3.7): pick a sequence $\left\{a_{n}\right\}$ for which (3.13) does not hold but the Blaschke condition does; then rotate its terms so as to get an interpolating sequence $\left\{b_{n}\right\}$ with $\left|b_{n}\right|=\left|a_{n}\right|$.

A sequence satisfying (3.13) need not be interpolating; for example, consider $a_{n}=$ $1-n^{-2}$. Hence, the two classes are not comparable, but each of them contains the class of exponential sequences as a relatively small subclass.

How large can the exponent of integrability for $B^{\prime}$ be for an interpolating Blaschke product? We mentioned in $\S 1$ that the derivative of any Blaschke product necessarily belongs to all $A^{p}, 0<p<1$. Combining Naftalevich's theorem (Theorem 3.7) with Theorem 3.4, we obtain the following result.

Theorem 3.8. There exists an interpolating Blaschke product $B$ such that $B^{\prime} \notin$ $\bigcup_{1<p<\infty} A^{p}$.

Proof. Note that the sequence $a_{n}=1-\left(n \log ^{2} n\right)^{-1}$ satisfies the Blaschke condition. By Theorem 3.7, there exists a sequence $\left\{b_{n}\right\}$ which is interpolating for $H^{\infty}$ and such that $\left|b_{n}\right|=a_{n}$ for all $n$. Now, whenever $p>1$, we have

$$
\sum_{n=1}^{\infty}\left(1-\left|b_{n}\right|\right)^{2-p}=\sum_{n=1}^{\infty} \frac{1}{n^{2-p} \log ^{2(2-p)} n}=\infty,
$$

and the claim follows from Theorem 3.4 by choosing the interpolating Blaschke product with zeros $b_{n}$.

To the best of our knowledge, the existence of an interpolating Blaschke product whose derivative is not in $A^{1}$ remains an open question (and an interesting one). Note that this Bergman space coincides with the Banach space $B^{1 / 2}$ from the family of spaces studied by a number of authors (see, for example, [15]). This case has been a stumbling block for a long time and continues to be so.

\subsection{An approach via $Q_{p}$ spaces and Carleson measures}

As mentioned in $\S 1$, there is a connection between the membership of $B^{\prime}$ in Hardy and Bergman spaces on the one hand, and of $B$ in $Q_{p}$ spaces on the other hand.

When $0<p<\infty$, an analytic function $f$ in $\mathbb{D}$ belongs to the space $Q_{p}$ if

$$
\sup _{a \in \mathbb{D}} \int_{\mathbb{D}}\left|f^{\prime}(z)\right|^{2} g(z, a)^{p} \mathrm{~d} A(z)<\infty,
$$

where $g$ denotes the Green function for the disc given by

$$
g(z, a)=\log \left|\frac{1-\bar{a} z}{a-z}\right|, \quad z, a \in \mathbb{D}, z \neq a .
$$

The spaces $Q_{p}$ are conformally invariant. They have their origin in [35], where it was shown that $Q_{2}=\mathcal{B}$ (the Bloch space), and in [5], where this result was extended by 
showing that $Q_{p}=\mathcal{B}$ for all $p>1$. The space $Q_{1}$ coincides with BMOA. When $0<p<1$, $Q_{p}$ is a proper subspace of BMOA and has many interesting properties (see $[\mathbf{6}, \mathbf{1 6}, \mathbf{3 6}]$ ).

There are various characterizations of $Q_{p}$ spaces. The one that will be useful for us is expressed in terms of $p$-Carleson measures. Denote by $|I|$ the length of an interval $I$ on the unit circle $\mathbb{T}$. The Carleson square $S(I)$ is defined as

$$
S(I)=\left\{r \mathrm{e}^{\mathrm{i} \theta}: \mathrm{e}^{\mathrm{i} \theta} \in I, 1-\frac{|I|}{2 \pi} \leqslant r<1\right\} .
$$

Given a positive Borel measure $\mu$ in $\mathbb{D}$, we say that $\mu$ is a $p$-Carleson measure on $\mathbb{D}$ if there exists a positive constant $C$ such that

$$
\mu(S(I)) \leqslant C|I|^{p}, \quad \text { for every interval } I \subset \mathbb{T} .
$$

The special case $p=1$ yields the classical Carleson measures [13]. The following characterization of $Q_{p}$ spaces was obtained by Aulaskari et al. [6].

Theorem 3.9. Let $0<p<\infty$. A function $f$ holomorphic in $\mathbb{D}$ is a member of $Q_{p}$ if and only if the measure $\mu$ on $\mathbb{D}$ defined by $\mathrm{d} \mu_{p}(z)=\left(1-|z|^{2}\right)^{p}\left|f^{\prime}(z)\right|^{2} \mathrm{~d} A(z)$ is a p-Carleson measure.

Essén and Xiao [16] used this result to characterize the inner functions that belong to $Q_{p}$ spaces $(0<p<1)$. In particular, their result can be stated for Blaschke products as follows.

Theorem 3.10. Let $p \in(0,1)$ and let $B$ be a Blaschke product with zero sequence $\left\{a_{n}\right\}_{n=1}^{\infty}$. Then $B \in Q_{p}$ if and only if the measure $\mu_{p}$, defined as

$$
\mathrm{d} \mu_{p}=\sum_{n=1}^{\infty}\left(1-\left|a_{n}\right|^{2}\right)^{p} \delta_{a_{n}},
$$

is a $p$-Carleson measure. Here, as is usual, $\delta_{a_{n}}$ denotes the point mass at $a_{n}$.

The following concept was introduced in [12]. A sequence $\left\{\alpha_{n}\right\}_{n=1}^{\infty}$ such that $\alpha_{n} \geqslant 0$, $\alpha_{n} \geqslant \alpha_{n+1}, n=1,2, \ldots$, and $\lim _{n \rightarrow \infty} \alpha_{n}=0$ is said to be asymptotically concentrated if, for each $k=1,2, \ldots$, there exists an increasing infinite subsequence $n_{p}$ of positive integers that depends only on $k$, with the property that

$$
\lim _{p \rightarrow \infty} \frac{\alpha_{n_{p}}}{\alpha_{n_{p}+k}}=1 .
$$

Using this concept and Theorem 3.10, Danikas and Mouratides [12] obtained the following sufficient condition for membership of a Blaschke product in $\bigcap_{0<p<1} Q_{p}$ expressed only in terms of absolute values of its zeros.

Theorem 3.11. Let $B$ a Blaschke product. Let its zeros be arranged in increasing order of moduli: $\left|a_{n}\right| \leqslant\left|a_{n+1}\right|, n \geqslant 1$. Also, for each $n$, let $\alpha_{n}=1-\left|a_{n}\right|$. If the sequence $\left\{\alpha_{n}\right\}$ is not asymptotically concentrated, then $B \in \bigcap_{0<p<1} Q_{p}$. 
Using the lemma on p. 201 of [12], together with [13, Lemma 1, p. 150], Theorem 3.11 and Theorem 3.10, one can deduce the following result (see [28] for the details).

Theorem 3.12. Let $B$ be an interpolating Blaschke product whose zero sequence $\left\{a_{n}\right\}_{k=1}^{\infty}$ is contained in a Stolz angle. Then $B \in \bigcap_{0<p<1} Q_{p}$. Consequently, if $0<p<1$, then the measure $\mu_{p}$ given by

$$
\mathrm{d} \mu_{p}=\sum_{k=1}^{\infty}\left(1-\left|a_{n}\right|^{2}\right)^{p} \delta_{a_{n}}
$$

is a $p$-Carleson measure (and, hence, a finite measure).

This and Theorem 3.1 yield Theorem 2.6, as above, thus providing an alternative proof. Note that, of course, Theorem 3.12 can also be deduced using the result of Newman mentioned above and Theorem 3.10 .

\section{Note added in proof}

After the submission of this paper, Girela and Peláez proved that the exponent $\frac{3}{2}$ in Theorem 2.5 is sharp: [20, Theorem 1] asserts that the Blaschke product $B$ with zeros $a_{n}=1-1 /\left(n \log ^{2} n\right), n \geqslant 2$, has the property that $B^{\prime} \notin A^{3 / 2}$.

Acknowledgements. The authors thankfully acknowledge partial support from the following Spanish grants. D.G. was funded by MTM2004-00078 (MEC) and FEDER and FQM210 (Junta de Andalucía). J.A.P. was funded by BFM2003-00034 and 'El Programa Juan de la Cierva' (MEC) and FQM210 (Junta de Andalucía). D.V. was funded by BFM2003-07294-C02-01 (MCyT/MEC). All authors were funded by MTM2004-21420-E ('Acciones Complementarias', MEC).

\section{References}

1. P. AHERn, The mean modulus of the derivative of an inner function, Indiana Univ. Math. J. 28 (1979), 311-347.

2. P. Ahern, The Poisson integral of a singular measure, Can. J. Math. 35 (1983), 735-749.

3. P. Ahern AND D. Clark, On inner functions with $H^{p}$ derivative, Michigan Math. J. 21 (1974), 115-127.

4. P. Ahern and D. Clark, On inner functions with $B^{p}$ derivative, Michigan Math. J. 23 (1976), 107-118.

5. R. Aulaskari And P. Lappan, Criteria for an analytic function to be Bloch and a harmonic or meromorphic function to be normal, in Complex analysis and its applications, Pitman Research Notes in Mathematics, Volume 305, pp. 136-146 (Longman Scientific, 1994).

6. R. Aulaskari, D. A. Stegenga and J. XiaO, Some subclasses of BMOA and their characterizations in terms of Carleson measures, Rocky Mt. J. Math. 26 (1996), 485-506.

7. S. AXLER, Bergman spaces and their operators, in Surveys of some recent results in operator theory, 1 (ed. J. B. Conway and B. B. Morrel), Pitman Research Notes in Mathematics, Volume 171, pp. 1-50 (Longman, 1988).

8. L. Carleson, An interpolation problem for bounded analytic functions, Am. J. Math. 80 (1958), 921-930. 
9. G. W. Cochran, Random Blaschke products, Trans. Am. Math. Soc. 322 (1990), 731755 .

10. W. S. CoHn, On the $H^{p}$ classes of derivative of functions orthogonal to invariant subspaces, Michigan Math. J. 30 (1983), 221-229.

11. P. ColWell, Blaschke products: bounded analytic functions (University of Michigan Press, Ann Arbor, MI, 1985).

12. N. Danikas And C. Mouratides, Blaschke products in $Q_{p}$ spaces, Complex Variables 43 (2000), 199-209.

13. P. L. DuREn, Theory of $H^{p}$ spaces (Academic, 1970; reprinted Dover, 2000).

14. P. L. Duren And A. P. Schuster, Bergman spaces, Mathematical Surveys and Monographs, Volume 100 (American Mathematical Society, Providence, RI, 2004).

15. P. L. Duren And A. L. Shields, Properties of $H^{p}(0<p<1)$ and its continuing Banach space, Trans. Am. Math. Soc. 141 (1969), 255-262.

16. M. EssÉn AND J. XIAO, Some results on $Q_{p}$ spaces: $0<p<1$, J. Reine Angew. Math. 485 (1997), 173-195.

17. J. B. GarnetT, Bounded analytic functions (Academic, 1981).

18. D. Girela And C. GonzÁlez, Mean growth of the derivative of infinite Blaschke products, Complex Variables 45 (2001), 1-10.

19. D. Girela And J. A. Peláez, On the derivative of infinite Blaschke products, Illinois J. Math. 48 (2004), 121-130.

20. D. Girela And J. A. PelÁez, On the membership in Bergman spaces of the derivative of a Blaschke product with zeros in a Stolz domain, Can. Math. Bull. 49 (2006), 381-388.

21. H. Hedenmalm, B. Korenblum And K. Zhu, Theory of Bergman spaces, Graduate Texts in Mathematics, Volume 199 (Springer, 2000).

22. H. O. Kim, Derivatives of Blaschke products, Pac. J. Math. 114 (1984), 175-190.

23. M. A. KutBi, Integral means for the first derivative of Blaschke products, Kodai Math. J. 24 (2001), 86-97.

24. M. MatelJević, The isoperimetric inequality and some extremal problems in $H^{1}$, in Analytic functions, Lecture Notes in Mathematics, Volume 798, pp. 364-369 (Springer, 1980).

25. A. G. NAftalevič, On interpolation by functions of bounded characteristic, Vilniaus Valst. Univ. Mokslu Darbai. Mat. Fiz. Chem. Mokslu Ser. 5 (1956), 5-27 (in Russian).

26. D. J. Newman, Interpolation in $H^{\infty}$, Trans. Am. Math. Soc. 92 (1959), 501-507.

27. C. A. Nolder, An $L^{p}$ definition of interpolating Blaschke products, Proc. Am. Math. Soc. 128 (2000), 1799-1806.

28. J. A. Peláez, Contribuciones a la teoría de ciertos espacios de funciones analíticas, Doctoral thesis, Universidad de Málaga (2004, in Spanish).

29. G. Piranian, Bounded functions with large circular variation, Proc. Am. Math. Soc. 19 (1968), 1255-1257.

30. D. Protas, Blaschke products with derivative in $H^{p}$ and $B^{p}$, Michigan Math. J. 20 (1973), 393-396.

31. W. Rudin, The radial variation of analytic functions, Duke Math. J. 22 (1955), 235-242.

32. W. Rudin, Real and complex analysis (McGraw-Hill, 1974).

33. S. A. Vinogradov, Multiplication and division in the space of analytic functions with area integrable derivative, and in some related spaces, Zap. Nauchn. Semin. POMI 222 (1994), 45-77 (in Russian; Engl. transl. J. Math. Sci. 87, (1997), 3806-3827).

34. D. Vukotić, The isoperimetric inequality and a theorem of Hardy and Littlewood, Am. Math. Mon. 110 (2003), 532-536.

35. J. XIAO, Carleson measure, atomic decomposition and free interpolation from Bloch space, Ann. Acad. Sci. Fenn. A 19 (1994), 35-46.

36. J. XIAO, Holomorphic $Q$ classes, Lecture Notes in Mathematics, Volume 1767 (Springer, 2001). 